\title{
High Second-Order NLO Response Exhibited by the First Example of Polymeric Film Incorporating a Diimine-Dithiolate Square-Planar Complex: The [Ni(o-phen)(bdt)]
}

\author{
Luca Pilia, ${ }^{*}, \dagger$ Daniele Marinotto, ${ }^{\S}$ Maddalena Pizzotti, ${ }^{\S}$ Francesca Tessore, ${ }^{\S}$ and Neil Robertson ${ }^{\ddagger}$ \\ ${ }^{\dagger}$ Dipartimento di Ingegneria Meccanica, Chimica e dei Materiali, Università di Cagliari, via Marengo 2, I09123 Cagliari, Italy \\ ${ }^{\ddagger}$ School of Chemistry and EaStChem, University of Edinburgh, King’s Buildings, West Mains Road, Edinburgh EH9 3FJ, United \\ Kingdom \\ ${ }^{\S}$ Dipartimento di Chimica, Università di Milano, Unità di Ricerca dell’INSTM, via C. Golgi 19, 20133 Milano, Italy
}

Supporting Information

ABSTRACT: A novel square-planar diimine-dithiolate nonlinear optical (NLO) chromophore, the complex $[\mathrm{Ni}(o-$ phen $)(\mathrm{bdt})](\mathbf{1})$, is reported in this paper $(o$-phen = dianion of 1,2-phenylendiamina; bdt $=1,2$-benzenedithiolate). This compound has been fully characterized by single-crystal X-ray diffraction, UV-vis-NIR spectroscopy, cyclic voltammetry, DFT, and TD-DFT calculations. 1 crystallizes in the $P_{21}$ space group and shows an almost planar molecule. The cyclic voltammetry measurements present an irreversible anodic peak at $0.87 \mathrm{~V}$ and reversible and quasi-reversible reduction waves at

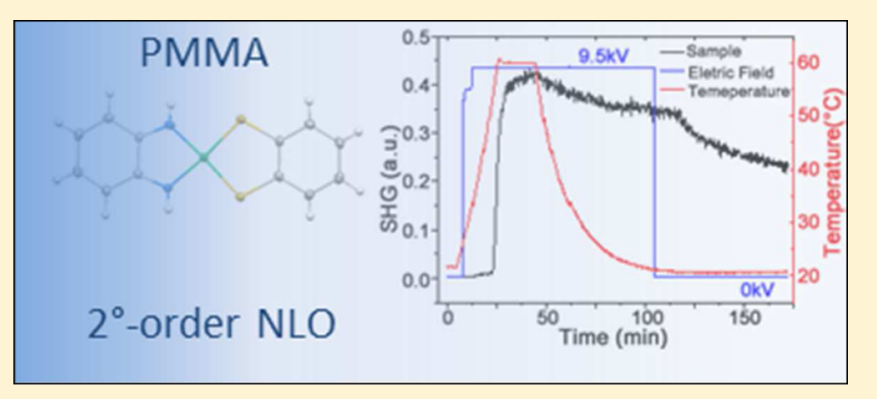
-0.35 and $-1.13 \mathrm{~V}$, respectively. A medium-intense $(\varepsilon=1.91$

$\times 10^{4} \mathrm{~mol}^{-1} \mathrm{dm}^{3} \mathrm{~cm}^{-1}$ ) absorption with a maximum at $728 \mathrm{~nm}$ appears in the UV-vis-NIR spectrum. The molecular secondorder NLO properties of 1 have been measured by the electric field induced second-harmonic generation technique in DMF solution, giving values of $-1000 \times 10^{-48}$ esu for $\mu \beta_{1.907}$ and $-356 \times 10^{-48}$ esu for $\mu \beta_{0}$. Furthermore, remarkable solid-state responses have been measured for polymeric films of 1 . Indeed, this complex has been embedded into poly(methyl methacrylate) poled films showing high NLO response $\left(d_{33}=1.90 \pm 0.38 \mathrm{pm} / \mathrm{V}\right)$, higher than those previously reported for similar push-pull metal complexes. Moreover, 1 represents the first example of a diimine-dithiolate chromophore incorporated into a NLO-active film.

\section{INTRODUCTION}

Since the development of lasers several nonlinear optical (NLO) effects, such as second- and third-harmonic generation, stimulated scattering, self-focusing, etc., have been discovered or experimentally proved. ${ }^{1-3}$ Materials showing NLO properties have been extensively investigated in the last decades because of their potential use in telecommunication technologies and optoelectronic and photonic devices. ${ }^{1-10}$

Molecule-based materials exhibiting second-order NLO properties ( $\beta$ and $\chi^{(2)}$ at molecular and bulk level, respectively) have received great attention because of their large and fast NLO response and easy processability. ${ }^{4-10}$ So far, different classes of molecular NLO chromophores have been reported in the literature, both "full" organic ${ }^{3,11-14}$ and inorganic. In particular, the latter class comprises push-pull and octupolar complexes of different transition metals showing remarkable $\beta$ values. $^{5-10,15,16}$ Moreover, different types of reversible switching of the NLO property have been achieved in the case of several complexes. ${ }^{17}$

For symmetry reasons, only materials without inversion center exhibit second-order NLO effects $\left(\beta\right.$ and $\left.\chi^{(2)} \neq 0\right)$.
Among the molecular second-order NLO chromophores, many square-planar push-pull complexes showing remarkable values of $\beta$ have been reported, in particular dithionedithiolate $^{18-24}$ and diimine-dithiolate s,25-31 $^{5}$ compounds. These complexes are formed by a metal ion bound to two ligands with different electron-withdrawing capability. Indeed, the push ligand presents electron donor groups, which raise the energy level of its orbitals, whereas those of the pull ligand are stabilized by the electron-withdrawing effect due to its substituents. In general, the pull ligand contributes more to the HOMO while the LUMO is predominantly formed by the push ligand (HOMO = highest occupied molecular orbital, LUMO = lowest unoccupied molecular orbital). Because of these features, the solvatochromic HOMO-LUMO transition, which falls in the visible region with a medium molar absorption coefficient and plays a crucial role in the secondharmonic generation (SHG, $\beta$ at the molecular level), has a charge transfer (CT) character. ${ }^{18-31}$ By choosing properly the

Received: May 2, 2016

Revised: July 25, 2016

Published: August 4, 2016 
substituents at the periphery of the ligands, it is possible to tune the dipole moment of the molecule and the energy of the CT transition, as well as the electronic structure of the frontier orbitals (FOs).

Although the molecules of push-pull complexes are noncentrosymmetric $(\beta \neq 0)$, very often they crystallize in a centrosymmetric space group preventing the second-order effects from occurring at the bulk level $\left(\chi^{(2)}=0\right)$. The centrosymmetric arrangement in diimine-dithiolate and dithione-dithiolate complexes is favored by their intrinsic polarity, due to the different electronic properties of the two ligands. Moreover, even if the crystal packing is noncentrosymmetric, if the molecules show a pseudocentrosymmetric packing, the $\chi^{(2)}$ value is close to zero. ${ }^{32}$ For these reasons, no dithiolate containing square-planar push-pull complexes showing solid-state second-order NLO properties have been reported to date. However, because of the paramount importance of NLO response in the solid state for technological applications, in order to overcome this problem, the strategy to incorporate NLO-active molecules in polymeric $^{33-37}$ or hybrid organic-inorganic ${ }^{38,39}$ poled films has been developed and successfully applied to pure organic $^{33,34,38,39}$ and organometallic ${ }^{35-37}$ chromophores.

Surprisingly, despite high first hyperpolarizability values exhibited by the dithiolate square-planar unsymmetrical complexes, NLO studies in bulk of films containing any of these compounds have not been reported in the literature so far, although platinum diamine-dithiolate complexes [Pt( $\mathrm{R}_{2}$ bipy) (dmipi)] (dmipi =4,5-dimercapto-1,3-dithiol-2-propargylimino and $\mathrm{R}=\mathrm{H}, \mathrm{t}-\mathrm{Bu}$ ) have been covalently bound to hydrogen-terminated silicon (100) surfaces. ${ }^{40}$ In this paper we report the first example of a film incorporating a chromophore of this class of molecules: the novel $[\mathrm{Ni}(o$-phen $)(\mathrm{bdt})]$ (1) complex $(o$-phen $=$ dianion of 1,2-phenylendiamina; bdt $=1,2$ benzenedithiolate). This compound has been embedded into a poly(methyl methacrylate) (PMMA) matrix showing remarkably high NLO response. Moreover, 1 has been fully characterized by single-crystal X-ray diffraction, UV-vis-NIR spectroscopy, cyclic voltammetry, DFT, and TD-DFT calculations. The second-order NLO responses of 1 were also measured in solution by the electric field induced secondharmonic (EFISH) generation technique.

\section{EXPERIMENTAL SECTION}

All the reagents and solvents were purchased from Aldrich and used without further purification. The complex $\left[\mathrm{Ni}(o \text {-phen })_{2}\right]$ was prepared as previously described, ${ }^{41}$ whereas $\left[\mathrm{Ni}(\mathrm{bdt})_{2}\right]$ was synthesized following the same procedure used to prepared the analogue complex ${ }^{42}$ of 3,5-ditertiarybutyl-1,2-benzenedithiolate ligand (yield 80\%).

Preparation. [Ni(o-phen)(bdt)] (1). Synthesis. First, 210 $\mathrm{mg}(0.62 \mathrm{mmol})$ of $\left[\mathrm{Ni}(\mathrm{bdt})_{2}\right]$ in $40 \mathrm{~mL}$ of warm $\mathrm{DMF}$, green solution, was added dropwise to a warm solution of $[\mathrm{Ni}(o$ phen $\left.)_{2}\right](80 \mathrm{mg}, 0.30 \mathrm{mmol})$ in the same solvent $(40 \mathrm{~mL})$. The resulting solution was refluxed $4 \mathrm{~h}$ and then allowed to cool to room temperature (R.T.); after $18 \mathrm{~h}$ of stirring at R.T., the solvent was rotoevaporated and the crude product was partially dissolved with acetone, giving a purple solution. A green-brown solid (49 $\mathrm{mg}$ ), insoluble in acetone, was separated from the solution by centrifugation. The acetone solution was concentrated until a precipitate appeared and, after the addition of $30 \mathrm{~mL}$ of $n$-hexane, the evaporation of the acetone was completed. A purple microcrystalline solid was collected by centrifugation, washed three times with $n$-hexane, and air-dried (yield $128 \mathrm{mg}, 0.42 \mathrm{mmol} ; 70 \%$ ). Analytical results are in accordance with the formula $[\mathrm{Ni}(\mathrm{bdt})(o$-phen $)]$. Elemental Analysis: calculated for $\mathrm{C}_{12} \mathrm{H}_{10} \mathrm{~N}_{2} \mathrm{~S}_{2} \mathrm{Ni}$ (305.04): C 47.25, H 3.30, N 9.18; found: C 47.34, H 3.36, N 9.21. MS (EI): $m / z$ (\%) $304.0(100.00 \%)[\mathrm{M}+]$. UV-vis [in DMF; $\lambda, \mathrm{nm}\left(\varepsilon, \mathrm{dm}^{3}\right.$ $\left.\left.\mathrm{mol}^{-1} \mathrm{~cm}^{-1}\right)\right]: 728\left(1.91 \times 10^{4}\right) ; 499\left(4.04 \times 10^{3}\right) ; 355$, sh. FT-IR (KBr): $\nu_{\max } / \mathrm{cm}^{-1} 3260(\mathrm{~m}) ; 3090(\mathrm{vw}) ; 3073(\mathrm{vw})$; 3043(w); 3007(vw); 1600(mw); 1564(w); 1524(w) 1515(mw); 1453(m); 1419(mw); 1382(s); 1367(mw); 1321(w); 1283(mw); 1238(w); 1204(w); 1149(w); 1130(w); $1123(\mathrm{w}) ; 1096(\mathrm{w}) ; \quad 802(\mathrm{vs}) ; 782(\mathrm{mw}) ; \quad 768(\mathrm{~m}) ; 726(\mathrm{~s})$; 680(mw); 623(m); 560(w); 450(m).

Elemental analyses were performed with a Carlo Erba CE1108 Elemental Analyzer. MS were carried out on ThermoElectron MAT 900 by electron impact ionization technique. The UV-Vis-NIR spectra were recorded with a Jasco V-670 spectrophotometer using a quartz cell of path length $1 \mathrm{~cm}$. IR measurements $\left(4000-400 \mathrm{~cm}^{-1}\right)$ were performed with a spectrophotometer FT-IR Bruker Tensor27 on $\mathrm{KBr}$ pellets. Cyclic voltammograms were recorded on an $\mu$ AUTOLAB Type III potentiostat, driven by the GPES electrochemical software, using a conventional three-electrode cell consisting of a platinum wire working electrode, a platinum wire as counter-electrode, and $\mathrm{Ag} / \mathrm{AgCl}$ in saturated $\mathrm{LiCl}$ ethanol solution as reference electrode. The experiments were performed at R.T., in dry and argon-degassed DMF containing $0.1 \mathrm{~mol} \mathrm{dm}^{-3} \mathrm{Bu}_{4} \mathrm{NPF}_{6}$ as supporting electrolyte, at $25-200$ $\mathrm{mV} \mathrm{s}^{-1}$ scan rate. Data are quoted against $\mathrm{Ag} / \mathrm{AgCl}$; the $E_{1 / 2}$ for ferrocene/ferrocenium couple (internal standard) is $+0.54 \mathrm{~V}$ under the above-reported conditions. Single-crystal data were collected at $120 \mathrm{~K}$ on an Agilent Technologies SuperNova diffractometer $\left(\lambda=\mathrm{CuK}_{\alpha}, 1.54184 \AA\right)$. Absorption corrections were done by multiscan methods using CrysAlisPro; ${ }^{43}$ the structure was solved using Patterson direct charge flipping methods (Superflip) ${ }^{44}$ and refined on $F^{2}$ with full-matrix squares (SHELXTL). ${ }^{45}$ Non-hydrogen atoms were refined anisotropically. All $\mathrm{H}$ atoms were located in a difference Fourier map and freely refined. Graphical material was prepared with ORTEP $^{46}$ and Mercury ${ }^{47}$ programs. CCDC 1449051 contains the supplementary crystallographic data for this paper.

DFT Calculations. Density Functional Theory (DFT) $)^{48}$ studies on the ground-state electronic structure of 1 were performed using the GAUSSIAN $09^{49}$ software package. B3LYP, ${ }^{50,51}$ CAM-B3LYP ${ }^{52}$ (see Supporting Information), and $\mathrm{PBE} 1 \mathrm{PBE}^{53}$ (Supporting Information) were used throughout this investigation as functionals; moreover, the valence triple- $\zeta 6-311+G(d, p)^{54,55}$ was employed as basis set for all atoms. More details are given in the Supporting Information.

NLO Characterization. Solution. The NLO response of $\mathbf{1}$ in solution was measured by EFISH experiments ${ }^{56,57}$ as described in ref 30 , using a freshly prepared $10^{-3} \mathrm{M}$ solution of the NLOphore in DMF (see Supporting Information for more details). Solid-state SHG by Kurtz-Perry measurements ${ }^{58}$ were performed as reported in ref 59 and are summarized in the Supporting Information.

Films. Details on materials, methods, and preparation of the films of $\mathbf{1}$ in poly(methyl methacrylate) (PMMA), along with the Corona Poling setup, are reported in the Supporting Information

Maker Fringe Measurement. The absolute second-order NLO coefficient matrix values $d_{i j}$ were obtained by following the standard Maker fringe technique ${ }^{60}$ as described in ref 61 . 
Scheme 1. Schematic Depiction of the Synthesis of 1

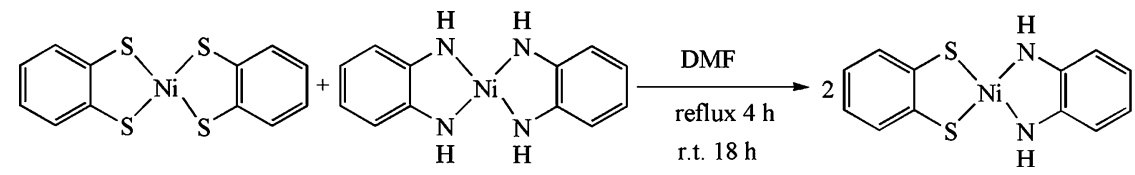

\section{RESULTS AND DISCUSSION}

Complex 1 has been synthesized in a good yield by refluxing $\left[\mathrm{Ni}(\text { bdt })_{2}\right]$ and $\left[\mathrm{Ni}(o \text {-phen })_{2}\right]$ in DMF as shown in Scheme 1 .

Structural Results. Blade-shaped crystals suitable for single-crystal X-ray diffraction analysis were obtained by slow evaporation of a dichloromethane solution. A summary of data collection and structure refinement is reported in Table 1.

\section{Table 1. X-ray Crystallographic Data for 1}

$\begin{array}{ll}\text { empirical formula } & \mathrm{C}_{12} \mathrm{H}_{10} \mathrm{~N}_{2} \mathrm{NiS}_{2} \\ \text { formula weight } & 305.05 \\ \text { color, habit } & \text { blue-purple } \\ \text { crystal size, mm } & 0.40 \times 0.06 \times 0.04 \text {, needle } \\ \text { crystal system } & \text { monoclinic } \\ \text { space group } & \mathrm{P} 21 \\ a, \AA & 6.0974(1) \\ b, \AA & 7.3037(1) \\ c, \AA & 12.9730(2) \\ \beta, \text { deg } & 90.681(1) \\ V, \AA^{3} & 577.70(2) \AA^{3} \\ Z & 2 \\ T, \mathrm{~K} & 120 \\ \rho(\text { calc }), \mathrm{Mg} / \mathrm{m}^{3} & 1.754 \\ \mu, \mathrm{mm}-1 & 5.585 \\ \theta \text { range, deg } & 3.4-73.8 \\ \text { no.of rflcn } / \text { unique } & 4717 / 2117 \\ \text { GooF } & 1.058 \\ \mathrm{R}^{a} & 0.0171 \\ \text { wR2 } & \\ & \end{array}$

${ }^{a} \mathrm{R} 1=\Sigma|| \mathrm{Fo}|-| \mathrm{Fc} \| / \Sigma \mid \mathrm{Fol} .{ }^{b} \mathrm{wR} 2=\left[\Sigma\left[w\left(\mathrm{Fo}^{2}-\mathrm{Fc}^{2}\right)^{2}\right] / \Sigma\left[w\left(\mathrm{Fo}^{2}\right)^{2}\right]\right]-$ $1 / 2, w=1 /\left[\sigma^{2}\left(\mathrm{Fo}^{2}\right)+(a P)^{2}+b P\right]$, where $P=\left[\max \left(\mathrm{Fo}^{2}, 0\right)+2 \mathrm{Fc}^{2}\right] / 3$

[Ni(bdt)(o-phen) $]$ crystallizes in the $P_{21}$ space group, which is a noncentrosymmetric group. As discussed in the Introduction, this feature is an essential requirement for a material to exhibit solid-state second-order NLO properties $\left(\chi^{(2)} \neq 0\right)$. Unfortunately, in this complex the second-order NLO susceptibility is close to zero (vide infra). A representation of a molecule of $\mathbf{1}$ is reported in Figure 1A; the molecule is almost planar. Indeed all non- $\mathrm{H}$ atoms are coplanar to within $0.05 \AA$, and the angles between the plane determined by $\mathrm{S}_{2} \mathrm{NiN}_{2}$ atoms and those of the benzene rings of the dithiolene and diamine moieties are $5.06^{\circ}$ and $2.35^{\circ}$, respectively (Figure S1). The bond distances (see Table 2) are similar to those reported in the literature for other nickel complexes of the same ligands. ${ }^{42,62-64}$ The $\mathrm{C}-\mathrm{N}$ bond distances are 1.313 and $1.315 \AA$; these values are smaller than those found for the free ligand $(1.406-1.408 \AA)^{65}$ and also than those reported for other diimine-dithiolate complexes (1.350-1.368 $\AA) .^{30,31,66}$ These data strongly support a behavior as diimine of the $o$-phen ligand in complex $\mathbf{1}$.

Electrochemical Studies. The DMF cyclic voltammogram of 1 (Figure 2) presents an irreversible anodic peak at $0.87 \mathrm{~V}(0$ $\rightarrow+1)$ and reversible $\left(i_{\mathrm{c}} / i_{\mathrm{a}} \cong 1\right)$ and quasi-reversible reduction waves at $-0.35(0 \rightleftharpoons-1)$ and $-1.13 \mathrm{~V}(-1 \rightarrow-2)$, respectively. These values show that the electrochemical
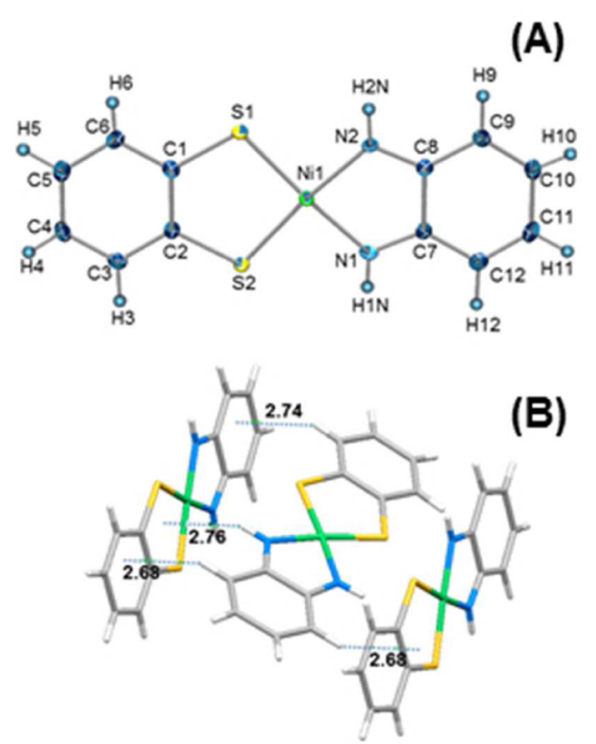

Figure 1. (A) Ortep diagram of $[\mathrm{Ni}(\mathrm{bdt})(o-\mathrm{phen})]$ (1) with atom labeling and thermal ellipsoids drawn at the $50 \%$ probability level. (B) Close $\mathrm{C}-\mathrm{H} \cdots \pi$ and $\mathrm{N}-\mathrm{H} \cdots \pi$ interactions between adjacent molecules.

Table 2. Comparison of Experimental and B3LYP Calculated Bond Lengths $(\AA)$ and Angles (deg) for 1

\begin{tabular}{cccc}
$\mathrm{Ni}(1)-\mathrm{S}(1)$ & $2.1226(5)$ & $\mathrm{S}(1)-\mathrm{Ni}(1)-\mathrm{S}(2)$ & $92.60(2) / 92.66$ \\
& $/ 2.152$ & & \\
$\mathrm{Ni}(1)-\mathrm{S}(2)$ & $2.1175(5)$ & $\mathrm{S}(1)-\mathrm{Ni}(1)-\mathrm{N}(2)$ & $92.83(5) / 92.22$ \\
& $/ 2.152$ & & \\
$\mathrm{Ni}(1)-\mathrm{N}(1)$ & $1.863(2)$ & $\mathrm{S}(1)-\mathrm{Ni}(1)-\mathrm{N}(1)$ & $176.03(5)$ \\
& $/ 1.878$ & & $/ 175.12$ \\
$\mathrm{Ni}(1)-\mathrm{N}(2)$ & $1.873(2)$ & $\mathrm{S}(2)-\mathrm{Ni}(1)-\mathrm{N}(1)$ & $91.36(5) / 92.23$ \\
& $/ 1.878$ & & \\
$\mathrm{C}(1)-\mathrm{S}(1)$ & $1.747(2)$ & $\mathrm{N}(1)-\mathrm{Ni}(1)-\mathrm{N}(2)$ & $83.22(7) / 82.89$ \\
& $/ 1.748$ & & \\
$\mathrm{C}(2)-\mathrm{S}(2)$ & $1.744(2)$ & $\mathrm{Ni}(1)-\mathrm{S}(1)-\mathrm{C}(1)$ & $105.06(6)$ \\
$\mathrm{C}(1)-\mathrm{C}(2)$ & 1.1 .748 & & $/ 104.56$ \\
& $1.405(3)$ & $\mathrm{Ni}(1)-\mathrm{N}(1)-\mathrm{C}(7)$ & $115.9(1) / 115.7$ \\
$\mathrm{~N}(1)-\mathrm{C}(7)$ & $1.313(2)$ & $\mathrm{S}(1)-\mathrm{C}(1)-\mathrm{C}(2)$ & $118.6(1) / 119.1$ \\
$\mathrm{~N}(2)-\mathrm{C}(8)$ & $/ 1.325$ & & \\
& $1.315(2)$ & $\mathrm{N}(1)-\mathrm{C}(7)-\mathrm{C}(8)$ & $112.7(2) / 112.8$ \\
$\mathrm{C}(7)-\mathrm{C}(8)$ & 1.325 & & \\
& $1.465(3)$ & & \\
\hline
\end{tabular}

properties of $\mathbf{1}$ are in between those of the corresponding homoleptic complexes. Indeed, in the case of $\left[\mathrm{Ni}(o \text {-phen })_{2}\right]$, the oxidation and the first reduction processes are both reversible and fall at 0.18 and $-0.83 \mathrm{~V}$, ${ }^{67}$ whereas for $\left[\mathrm{Ni}(\mathrm{bdt})_{2}\right]^{-}$the peaks corresponding to the same processes fall at 1.40 and $0.17 \mathrm{~V}$, respectively. ${ }^{68}$

UV-Vis-NIR Measurements. The UV-Vis-NIR spectrum of $\mathbf{1}$ is shown in Figure 3a. It presents a shoulder at 355 $\mathrm{nm}$ and a medium and a medium-intense absorption $\left(\mathrm{dm}^{3}\right.$ $\left.\mathrm{mol}^{-1} \mathrm{~cm}^{-1}\right)$ at $499\left(4.04 \times 10^{3}\right)$ and $728 \mathrm{~nm}\left(1.91 \times 10^{4}\right)$, respectively. The latter band, which is the most important in 

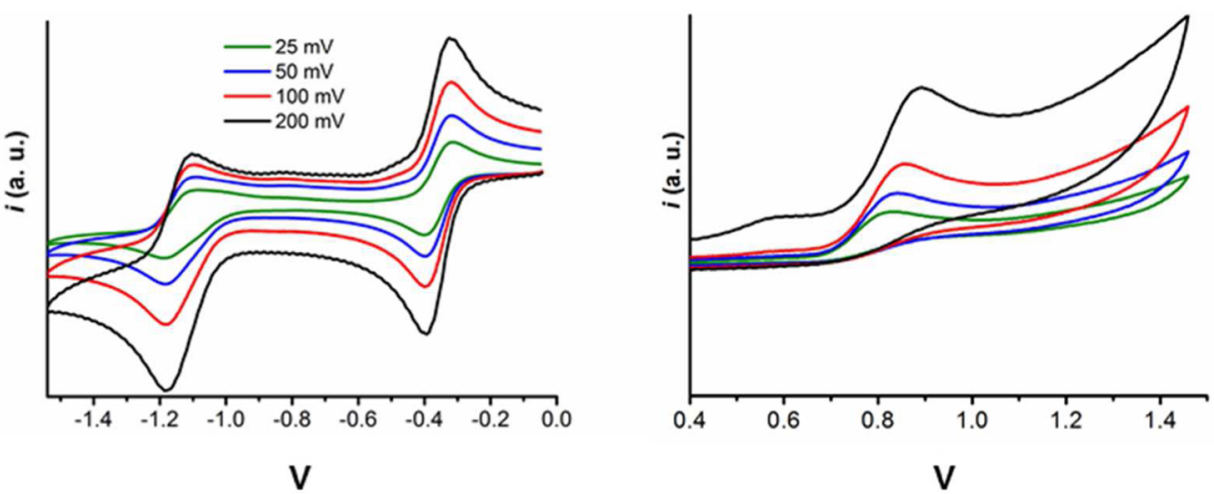

Figure 2. Cyclic voltammograms of $\mathbf{1}$ recorded at different scan rates in a DMF solution.
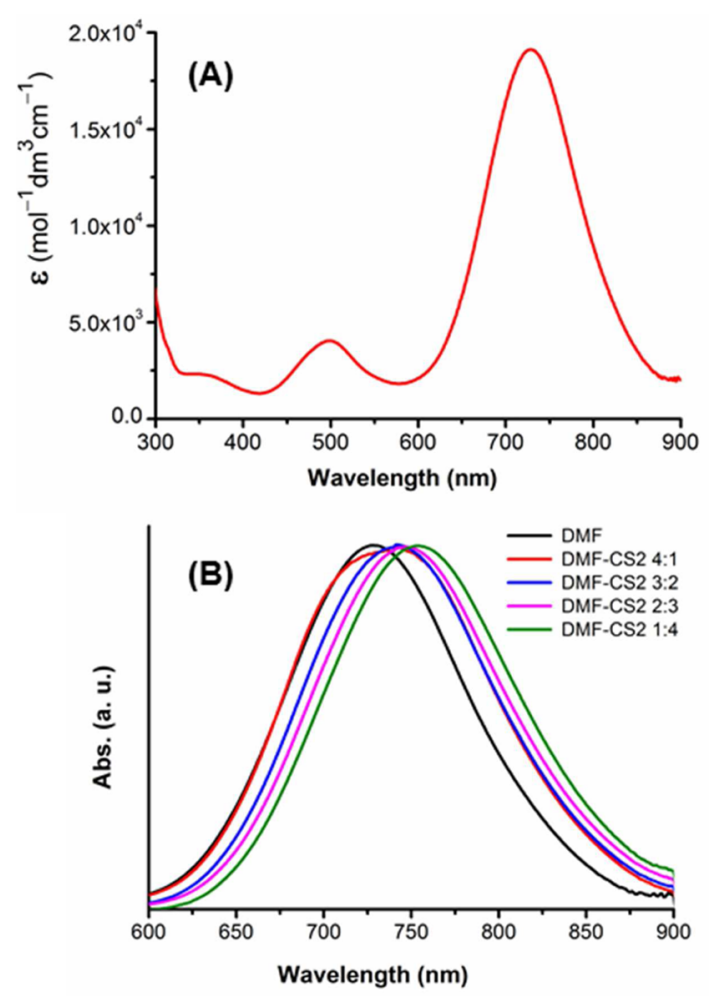

Figure 3. (A) UV-Vis-NIR spectrum of 1 in DMF. (B) Solvatochromic effect of complex 1 in $\mathrm{DMF} / \mathrm{CS}_{2}$ mixtures with different ratios.

NLO property generation, corresponds to a HOMO-LUMO transition and shows a moderate negative (hypsochromic shift) solvatochromic effect (Figure 3b), confirming the CT character of this transition in agreement with the computational results (vide infra). In 1 the solvatochromic effect is remarkably smaller $(26 \mathrm{~nm})$ than those found for other diiminedithiolate $^{30,31,69}$ and dithione-dithiolate ${ }^{20,21}$ complexes (80$140 \mathrm{~nm}$ ). Furthermore, the energies of the maxima of the solvatochromic bands present a linear behavior vs solvent polarity parameters $\left(r^{2}>0.939\right)$ as proposed by Eisenberg for several Pt-diimine-dithiolate compounds ${ }^{69}$ (Figure S6a); as expected, the solvatochromic shift, obtained from the plot's slope, is smaller (0.074) than those reported in ref 69 and for other push-pull compounds ${ }^{20,21,30,31}$ in agreement with the smaller solvatochromic effect exhibited by 1 . A similar trend $\left(r^{2}\right.$ $>0.938$ ) is also observed when Reichardt's solvent polarity parameters are used (Figure S6b). ${ }^{70}$
Computational Studies. The electronic structure of $\mathbf{1}$ has been investigated by DFT calculations at the B3LYP/6$311+\mathrm{G}(\mathrm{d}, \mathrm{p})$ level of theory. The optimized geometry in the gas phase is depicted in Figure S7. The calculated structure is planar, and both bond distances and angles are in very good agreement with the crystallographic data (see Table 2). The molecular orbitals of $\mathbf{1}$ calculated in the gas phase are shown in Figure 4. The frontier orbitals (FOs) and the HOMO-1

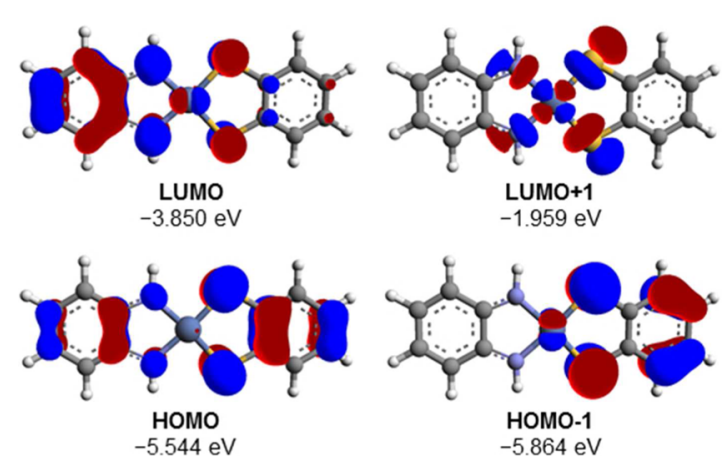

Figure 4. Molecular orbitals for 1 (contour value 0.040).

present a $\pi$-symmetry, while the LUMO+1 is a $\sigma$-type orbital. The calculated fragment contributions to the FOs (see Table 3) show an electronic delocalization extended over the two ligands. Indeed, the contributions to the HOMO from the $o$ phen (push) and from the bdt (pull) are 30.1 and 67.1\%, respectively. In the LUMO orbital these contributions are $29.5 \%$ from the dithiolate and $58.5 \%$ from the diimine; moreover, also the metal's contribution to this MO is important (12.0\%). Although each FO has remarkable contributions from all the different fragments, the HOMO-LUMO transition has a significant CT character, more precisely, a ligand-to-mixedmetal-ligand CT character. The contributions calculated for $\mathbf{1}$ are significantly different from those found for other diiminedithiolate complexes ${ }^{30,31,66}(84.2-77.2 \%$ to the HOMO and $91.4-79.8 \%$ to the LUMO from the dithiolate and diimine ligand, respectively). The more extended delocalization presented by 1 seems to stabilize both the FOs in comparison with those of the above cited diimine-dithiolate compounds $(-5.54$ vs $-4.50 /-4.96 \mathrm{eV}$ and -3.85 vs $-2.93 /-3.39 \mathrm{eV}$ for HOMO and LUMO, respectively). These complexes show a solvatochromic effect stronger than $\mathbf{1}$, according to a more pronounced CT character presented by their HOMO-LUMO transitions. ${ }^{30,31,66}$ The smaller dipole moment $(\mu)$ calculated for $1(4.42 \mathrm{D})$ is also in agreement with the differences in the 
Table 3. Comparison of Calculated Fragment Contributions and Energy Levels of the FOs in Gas Phase and DMF of Complexes 1

\begin{tabular}{|c|c|c|c|c|c|c|c|c|}
\hline \multirow[b]{2}{*}{ phase } & \multicolumn{4}{|c|}{ НОМО } & \multicolumn{4}{|c|}{ LUMO } \\
\hline & energy $(\mathrm{eV})$ & bdt (\%) & diimine (\%) & $\mathrm{Ni}(\%)$ & energy $(\mathrm{eV})$ & bdt (\%) & diimine (\%) & $\mathrm{Ni}(\%)$ \\
\hline gas & -5.544 & 67.1 & 30.1 & 2.8 & -3.852 & 29.5 & 58.5 & 12.0 \\
\hline DMF & -5.737 & 77.9 & 17.8 & 4.3 & -3.891 & 19.9 & 69.5 & 10.6 \\
\hline
\end{tabular}

electronic structures observed between this compound and other diimine-dithiolate complexes $(\mu=8.07-11.15 \mathrm{D}) .^{30,31}$

With the aim to evaluate the effect of the solvation on the electronic structure of $\mathbf{1}$, DFT calculations in DMF, modeled by the CPCM, have been done. These studies show that, in comparison with the gas phase, the solvation induces a greater polarization in the molecule, as evidenced from the composition of the frontier orbitals (Table 3) and the calculated $\mu(10.22 \mathrm{D})$. Moreover, the solvation also affects the energy of the orbitals (Table 3) although not in the same manner; indeed it stabilizes the HOMO more than the LUMO increasing the energy gap. The charge redistribution associated with the solvent-induced polarization is evident by comparison of the electrostatic potentials, mapped on electron density isosurfaces, calculated for $\mathbf{1}$ in the gas phase and DMF (Figure S8). Indeed, the solvation affects the charge separation between the two ligands, increasing the negative charge over the dithiolate one. Time-dependent DFT calculations have been performed in order to investigate the electronic transitions of 1 in both gas phase and DMF. The calculated electronic spectrum in DMF is in very good agreement with the experimental one, measured in the same solvent (Figure S9). The transition at $728 \mathrm{~nm}$ presents a molar extinction coefficient $\left(1.91 \times 10^{4} \mathrm{dm}^{3}\right.$ mol cm$~^{-1}$ ) remarkably higher than those found for other compounds of the same class $\left(2.50 \times 10^{3}-6.00 \times 10^{3} \mathrm{dm}^{3} \mathrm{~mol}\right.$ $\left.\mathrm{cm}^{-1}\right) .{ }^{30,31,66}$ This finding is strongly in agreement with the calculated values of the oscillator strength $(f)$ which is related to the intensity of the electronic transition. Indeed, $f$ calculated in DMF is 0.433 for 1 , whereas it ranges between 0.130 and 0.155 for the other complexes. ${ }^{30,31}$

NLO Properties. The second-order NLO properties of 1 have been investigated by the EFISH method, ${ }^{56}$ with measurements performed in DMF using an incident laser beam at $1.907 \mu \mathrm{m}$ of wavelength. EFISH experiments give a measure of the scalar product $\mu \beta_{\lambda}$, where $\mu$ is the molecular dipole moment and $\beta_{\lambda}$ the vector part of the quadratic hyperpolarizability tensor; $\beta_{\lambda}$ depends on the frequency of the incident light. The static quadratic hyperpolarizability $\beta_{0}$ is the extrapolated value to zero frequency and can be calculated taking into account the wavelength of the maximum of the absorption of the CT transition of the chromophore. The NLO response of 1 was $-1000 \times 10^{-48}$ esu for $\mu \beta_{\lambda}$ which corresponds to a value of $-356 \times 10^{-48}$ esu for $\mu \beta_{0}$. The negative value is in agreement with the observed negative solvatochromism. More notably, despite the small solvatochromic effect exhibited by $\mathbf{1}$ in comparison with those of the above-cited diimine-dithiolate complexes, ${ }^{30,31}$ the NLO response of $\mathbf{1}$ is quite high. For example, it appears higher than those reported for the first examples of $\mathrm{Ni}(\mathrm{II})$ diiminedithiolate complexes ${ }^{28}$ and comparable to that reported by some of us for $\left[\mathrm{Ni}\left(4,4^{\prime}\right.\right.$-dimethylcarboxy-bpy)(bdt)], carrying an electron-withdrawing group. ${ }^{30}$ An explanation of this finding can be obtained taking into account a simplified model, the two-state one $\mathrm{fl}^{71-74}$ (eq 1$)$, which provides a relationship between $\beta_{0}, \lambda_{\max }$ (the maximum of the HOMO-LUMO transition), $f$, and the difference between the ground- and excited-state dipole moments $\left(\Delta \mu_{g e}\right)$.

$$
\beta_{0}=1.617 \lambda_{\text {max }}^{3} f \cdot \Delta \mu_{\mathrm{ge}}
$$

The two-state model, even though is a simplification, furnishes a qualitatively good correlation between the characteristic of the molecules and their second-order NLO properties, as demonstrated for other push-pull chromophores both organic $^{11}$ and inorganic. ${ }^{18-21,30,66,75,76}$ In the case of complex 1, the high value of the oscillator strength (see above) seems to play a crucial role in determining the NLO response of this complex, partially compensating the small difference of the dipole moment between the ground- and excited-state $\left(\Delta \mu_{\mathrm{ge}}\right.$ is related to the solvatochromic effect).

Because $\mathbf{1}$ crystallizes in a noncentrosymmetric space group, its second-order NLO properties have been investigated also at the solid state by Kurtz-Perry measurements. ${ }^{58}$ Unfortunately, despite its symmetry, $\mathbf{1}$ is incapable of SHG in the crystal form; this is probably due to the molecular packing with a pseudocentrosymmetric arrangement, which induces an almost null value for $\chi^{(2)} .32$

NLO Properties of the PMMA Films. The second-order NLO response of $\mathbf{1}$ in the solid state was achieved incorporating the chromophore into a poled PMMA film. Through the corona wire poling process at high temperature a noncentrosymmetric alignment of $\mathbf{1}$ in the PMMA film was reached, monitoring the SHG signal in situ during the poling and at R.T. after the electric field was switched off. The PMMA film was obtained by spin-coating. After the deposition of the film onto the glass substrate, the latter was rinsed with acetone on the side not containing the film, so to remove any residue left by the deposition phase. Before poling, the film was stored in the dark in air. Before and after poling, the electronic absorption spectrum of the 1/PMMA film was recorded (Figure S10). The shape of the main absorption band is very similar to that obtained in DMF solution (Figure 3), thus confirming that in the film there are no aggregates and that the PMMA matrix has no influence on the electronic structure of the NLO-phore. Comparing the spectra before and after poling, no perceivable Stark shift of the main absorption band is observed, while the decrease of the intensity of the band after poling can be due to dichroism effect, ${ }^{77,78}$ caused by the reorientation of the NLO-phore molecules in the polymeric matrix, given that no significant sublimation is detected during the poling experiments.

As already reported in the literature, ${ }^{79-81}$ a linear relationship between the $d_{33}$ coefficient (the figure of merit of the electrooptical properties) and the loading of a NLO chromophore in a PMMA film exists. However, in the present investigation a low loading (4\%) was chosen to prevent aggregations of the NLOphores. As a consequence, the $d_{33}$ values of the composite PMMA films investigated were very low.

For the poling experiments, a Q-switched Nd:YAG laser (producing a $1064 \mathrm{~nm}$ incident wavelength) was used, and the 


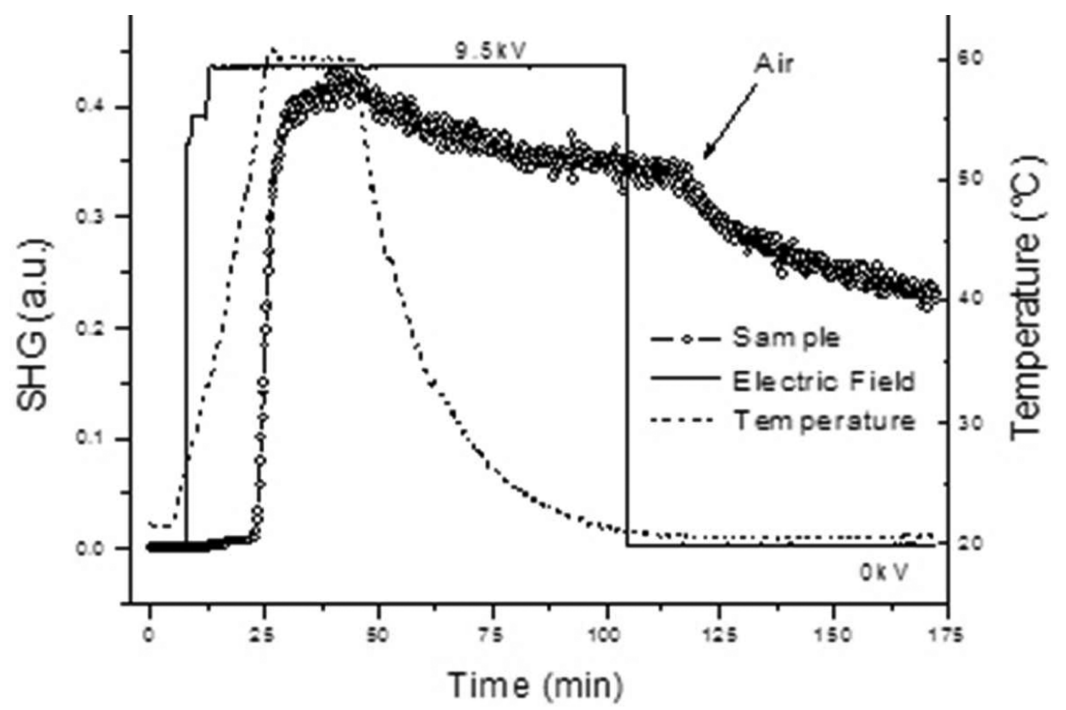

Figure 5. In situ corona wire poling dynamic of the SHG of the PMMA film containing the complex 1.

SHG signal was recorded in situ. The standard Maker fringe technique afforded a quantitative evaluation of the $d_{33}$ coefficient. The thickness of the poled films was measured by profilometry. Before poling, three vacuum-nitrogen cycles were applied to the film, placed inside a specially built drybox, so to lower the oxygen level as much as possible. In the poling process the ozone produced by the high electric field could quickly lead to a rapid and irreversible degradation of the film. For this reason performing the measure under nitrogen atmosphere is mandatory. The corona wire poling dynamic of the SHG of the investigated PMMA films is depicted in Figure 5. The SHG was negligible at room temperature. After application of the electric field, at the beginning the signal remained very low, but after $25 \mathrm{~min}$ it abruptly increased reaching its maximum value after $40 \mathrm{~min}$. After the plateau was reached, the temperature was decreased to $20^{\circ} \mathrm{C}$, and the loss of the SHG signal was only $20 \%$.

However, the most notable observation is that after removal of the applied electric field the SHG signal remained almost constant, showing a decrease only when the sample was moved from nitrogen to air atmosphere. At this stage, the decreasing of the SHG signal is expected due to the decrease of the local surface electric field. ${ }^{82}$ The second-order NLO coefficient matrix value $d_{33}$ for poled films was obtained by following the standard Maker fringe technique ${ }^{60}$ (Figures S11-S13), obtaining a remarkable value of $1.90 \pm 0.38 \mathrm{pm} / \mathrm{V}$, higher than those previously reported for similar push-pull metal complexes. $^{36,37}$ Moreover, it is noteworthy that these compounds show NLO responses at the molecular level $(\beta)$ higher than that exhibited by $1 .^{36,37}$

\section{CONCLUSIONS}

We prepared and characterized 1, a novel push-pull diiminedithiolate NLO chromophore. The X-ray data show a squareplanar molecular structure and bond distances in agreement with a depiction of this compound as a diimine-dithiolate complex. Spectroscopic and computational studies have shown that the optical absorption at $728 \mathrm{~nm}$ arises from a HOMOLUMO transition. Although with different contributions from the two ligands, both of the frontier orbitals are delocalized over all the molecule reducing the CT character of this transition, in agreement with the observed small solvatochromic effect. Despite these findings, 1 presents a good NLO response which can be explained taking into account the two-state model (eq 1 ) and the high value of $f$ related to the HOMO-LUMO electronic transition. The molecular second-order NLO properties of 1 were measured by EFISH method, giving values of -1000 and $-356 \times 10^{-48}$ esu for $\mu \beta_{1907}$ and $\mu \beta_{0}$, respectively. This complex has been also incorporated into PMMA poled films exhibiting remarkably high NLO response $\left(d_{33}=1.90 \pm 0.38 \mathrm{pm} / \mathrm{V}\right)$, higher than those showed by similar push-pull metal complexes. Notably, 1 represents the first example of diimine-dithiolate chromophore incorporated into a NLO-active film. Encouraged by these results, we will prepare films incorporating diimine-dithiolate complexes showing values of $\beta$ significantly higher than $1,{ }^{25-31}$ with the aim to achieve better solid-state NLO responses and to elucidate the structure-properties relationship.

\section{ASSOCIATED CONTENT}

\section{Supporting Information}

The Supporting Information is available free of charge on the ACS Publications website at DOI: 10.1021/acs.jpcc.6b04434.

Pictures of crystal structure of 1 (Figures S1-S5); list of van der Waals contacts (Table S1); solvatochromic peak vs solvent polarity parameters (Figure S6); DFToptimized geometry (Figure S7); comparison between experimental and calculated structural data (Table S2); electrostatic potential mapped on electron density isosurfaces (Figure S8); calculations with CAM-B3LYP and PBE1PBE as functionals; DMF calculated and experimental electronic spectra (Figure S9); frontier orbitals (Tables S3 and S4); HOMO and LUMO energies and compositions orbitals (Tables S5 and S6); electronic absorption spectra in the PMMA film (Figure S10); Maker fringe (Figures S11-S13); Hirshfeld surface (HS) analysis (Figures S14-S17) (PDF) Crystallographic data (CIF)

\section{AUTHOR INFORMATION}

\section{Corresponding Author}

*E-mail: pilialuc@unica.it. Phone: +39070675 5051. Fax: +39 0706755067. 


\section{Notes}

The authors declare no competing financial interest.

\section{ACKNOWLEDGMENTS}

L.P. thanks the FP-7 PEOPLE-Marie Curie Intra European Programme and gratefully acknowledges the Regione Autonoma della Sardegna for the financial support (P.O.R. SardegnaEuropean Social Fund 2007-2013-Line of Activity 1.3.1 "Avviso di chiamata per il finanziamento di Assegni di Ricerca”). We thank the EaStChem Research Computing Facility; authors are grateful to Dr. Gary Nichol and the University of Edinburgh Crystallography Service for collecting crystallographic data and solving the crystal structure.

\section{REFERENCES}

(1) He, G. S. Nonlinear Optics and Photonics; Oxford University Press: Oxford, 2015.

(2) Zyss, J. Nonlinear Optical Properties; Academic Press: New York, 1994.

(3) Chemla, D. S.; Zyss, J. Nonlinear Optical Properties of Organic Molecules and Crystals; Academic Press: New York, 1987; Vols. I and II..

(4) Marder, R. Materials for Nonlinear Optics, Chemical Perspectives; Stucky, G. D., Ed.; American Chemical Society: Washington, DC, 1991..

(5) Coe, B. J. Comprehensive Coordination Chemistry II; McCleverty, J. A., Meyer, T. J., Eds.; Elsevier Pergamon: Oxford, U.K., 2004; Vol. 9, pp 621-687.

(6) Di Bella, S. Second-Order Nonlinear Optical Properties of Transition Metal Complexes. Chem. Soc. Rev. 2001, 30, 355-366.

(7) Lacroix, P. Second-Order Optical Nonlinearities in Coordination Chemistry: The Case of Bis(salicylaldiminato)metal Schiff Base Complexes. Eur. J. Inorg. Chem. 2001, 2001, 339-348.

(8) Coe, B. J. Nonlinear Optical Properties of Matter: From Molecules to Condensed Phases; Papadopoulos, M. G., Sadlej, A. J., Leszczynski, J., Eds.; Springer: Dordrecht, Germany, 2006; Chapter 18, pp 571-608.

(9) Maury, O.; Le Bozec, H. Molecular Engineering of Octupolar NLO Molecules and Materials Based on Bipyridyl Metal Complexes. Acc. Chem. Res. 2005, 38, 691-704.

(10) Coe, B. J. Developing Iron and Ruthenium Complexes for Potential Nonlinear Optical Applications. Coord. Chem. Rev. 2013, 257, 1438-1458.

(11) Prasad, P. N.; Williams, D. J. Introduction to Nonlinear Optical Effects in Molecules and Polymers; John Wiley \& Sons, Inc.: New York, 1991.

(12) Marks, T. J.; Ratner, M. A. Design, Synthesis, and Properties of Molecule-Based Assemblies with Large Second-Order Optical Nonlinearities. Angew. Chem., Int. Ed. Engl. 1995, 34, 155-173.

(13) Ahlheim, M.; Barzoukas, M.; Bedworth, P. V.; Blanchard-Desce, M.; Fort, A.; Hu, Z.-Y.; Marder, S. R.; Perry, J. M.; Runser, C.; Staehelin, M.; et al. Chromophores with Strong Heterocyclic Acceptors: A Poled Polymer with a Large Electro-Optic Coefficient. Science 1996, 271, 335-337.

(14) Frattarelli, D.; Schiavo, M.; Facchetti, A.; Ratner, M. A.; Marks, T. J. Self-Assembly from the Gas-Phase: Design and Implementation of Small-Molecule Chromophore Precursors with Large Nonlinear Optical Responses. J. Am. Chem. Soc. 2009, 131, 12595-12612.

(15) Zyss, J. Molecular Engineering Implications of Rotational Invariance in Quadratic Nonlinear Optics: From Dipolar to Octupolar Molecules and Materials. J. Chem. Phys. 1993, 98, 6583-6599.

(16) Zyss, J.; Ledoux, I. Nonlinear Optics in Multipolar Media: Theory and Experiments. Chem. Rev. 1994, 94, 77-105.

(17) Coe, J. B.; Houbrechts, S.; Asselberghs, I.; Persoons, A. Efficient, Reversible Redox-Switching of Molecular First Hyperpolarizabilities in Ruthenium(II) Complexes Possessing Large Quadratic Optical Nonlinearities. Angew. Chem., Int. Ed. 1999, 38, 366-369.
(18) Chen, C.-T.; Liao, S.-Y.; Lin, K.-J.; Lai, L.-L. Syntheses, Charge Distribution, and Molecular Second-Order Nonlinear Optical Properties of Push-Pull Bisdithiolene Nickel Complexes. Adv. Mater. 1998, $10,334-338$.

(19) Pilia, L.; Artizzu, F.; Faulmann, C.; Mercuri, M. L.; Serpe, A.; Deplano, P. Square-Planar $\mathrm{d}^{8}$ Metal Push-Pull Dithiolene Complexes: Synthesis and Characterization of $\left[\mathrm{Pd}\left(\mathrm{Me}_{2} \mathrm{pipdt}\right)\right.$ (dmit)]. Inorg. Chem. Commun. 2009, 12, 490-493.

(20) Pilia, L.; Espa, D.; Barsella, A.; Fort, A.; Mitsopoulou, C. A.; Marchio, L.; Mercuri, M. L.; Serpe, A.; Makedonas, C.; Deplano, P. Combined Experimental and Theoretical Study on Redox-Active $\mathrm{d}^{8}$ Metal Dithione-Dithiolato Complexes Showing Molecular SecondOrder Nonlinear Optical Activity. Inorg. Chem. 2011, 50, 1001510027.

(21) Espa, D.; Pilia, L.; Marchiò, L.; Mercuri, M. L.; Serpe, A.; Barsella, A.; Fort, A.; Dalgleish, S. J.; Robertson, N.; Deplano, P. Redox-Switchable Chromophores Based on Metal (Ni, Pd, Pt) MixedLigand Dithiolene Complexes Showing Molecular Second-Order Nonlinear-Optical Activity. Inorg. Chem. 2011, 50, 2058-2060.

(22) Espa, D.; Pilia, L.; Marchiò, L.; Artizzu, F.; Mercuri, M. L.; Serpe, A.; Simão, D.; Almeida, M.; Pizzotti, M.; Tessore, F.; Deplano, P. Mixed-Ligand Pt(II) Dithione-Dithiolato Complexes: Influence of the Dicyanobenzodithiolato Ligand on the Second-Order NLO Properties. Dalton Trans. 2012, 41, 3485-3493.

(23) Espa, D.; Pilia, L.; Marchiò, L.; Pizzotti, M.; Robertson, N.; Tessore, F.; Mercuri, M. L.; Serpe, A.; Deplano, P. Electrochromic Second-Order NLO Chromophores Based on $\mathrm{M}^{\mathrm{II}}(\mathrm{M}=\mathrm{Ni}, \mathrm{Pd}, \mathrm{Pt})$ Complexes with Diselenolato-Dithione (Donor-Acceptor) Ligands. Dalton Trans. 2012, 41, 12106-12113.

(24) Espa, D.; Pilia, L.; Mercuri, M. L.; Serpe, A.; Marchiò, L.; Barsella, A.; Fort, A.; Makedonas, C.; Mitsopoulou, C. A.; Deplano, P. Role of the Acceptor in Tuning the Properties of Metal $[\mathrm{M}(\mathrm{II})=\mathrm{Ni}$, $\mathrm{Pd}, \mathrm{Pt}$ ] Dithiolato/Dithione (Donor/Acceptor) Second-Order Nonlinear Chromophores: Combined Experimental and Theoretical Studies. Inorg. Chem. 2014, 53, 1170-1183.

(25) Cummings, S. D.; Eisenberg, R. Dithiolene Chemistry: Synthesis, Properties, and Applications, Progress in Inorganic Chemistry; Stiefel, E. I., Ed.; Wiley, Chichester, U.K., 2004; Vol. 52, pp 315-367.

(26) Chen, C.-T.; Liao, S.-Y.; Lin, K.-J.; Lin, T.-Y. J.; Lia, L.-L.; Chen, C.-H. Molecular Second-Order Nonlinear Optical Properties of Unsymmetrical Nickel Complexes. MCLC S\&T, Sect. B: Nonlinear Opt. 1999, 22, 35-38.

(27) Base, K.; Tierney, M. T.; Fort, A.; Muller, J.; Grinstaff, M. On the Second-Order Nonlinear Optical Structure-Property Relationships of Metal Chromophores. Inorg. Chem. 1999, 38, 287-289.

(28) Cummings, S. D.; Cheng, L.-T.; Eisenberg, R. Metalloorganic Compounds for Nonlinear Optics: Molecular Hyperpolarizabilities of $\mathrm{M}$ (diimine) (dithiolate) Complexes $(\mathrm{M}=\mathrm{Pt}, \mathrm{Pd}, \mathrm{Ni})$. Chem. Mater. 1997, 9, 440-450.

(29) Chen, C.-T.; Lin, T.-Y. J.; Chen, C.-H.; Lin, K.-J. Molecular Nonlinear Optical Properties of Acceptors Substituted 2,2'-Bipyridine and 1,10-Phenathroline Complexes of Nickel Dithiolate. J. Chin. Chem. Soc. 2000, 47, 197-201.

(30) Pilia, L.; Pizzotti, M.; Tessore, F.; Robertson, N. NonlinearOptical Properties of $\alpha$-Diiminedithiolatonickel(II) Complexes Enhanced by Electron-Withdrawing Carboxyl Groups. Inorg. Chem. 2014, 53, 4517-4526.

(31) Pilia, L.; Pizzotti, M.; Tessore, F.; Robertson, N. Tuning the LUMO Energy of 1,10-Phenanthroline in $\alpha$-Diimine-Dithiolate Ni(II) Complex and Enhancement of Nonlinear Optical Properties. Inorg. Chim. Acta 2015, 430, 114-119.

(32) Faulmann, C.; Cassoux, P. Dithiolene Chemistry: Synthesis, Properties, and Applications, Progress in Inorganic Chemistry; Stiefel, E. I., Ed.; Wiley: Chichester, U.K., 2004; Vol. 52, pp 399-489.

(33) Dalton, L. R.; Sullivan, P. A.; Bale, D. H. Electric Field Poled Organic Electro-Optic Materials: State of the Art and Future Prospects. Chem. Rev. 2010, 110, 25-55.

(34) Dalton, L. R.; Harper, A. W.; Ghosn, R.; Steier, W. H.; Ziari, M.; Fetterman, H.; Shi, Y.; Mustacich, R. V.; Jen, K.-Y.; Shea, K. J. 
Synthesis and Processing of Improved Organic Second-Order Nonlinear Optical Materials for Applications in Photonics. Chem. Mater. 1995, 7, 1060-1081.

(35) Fillaut, J.-L.; Perruchon, J.; Blanchard, P.; Roncali, J.; Golhen, S.; Allain, M.; Migalsaka-Zalas, A.; Kityk, I. V.; Sahraoui, B. Design and Synthesis of Ruthenium Oligothienylacetylide Complexes. New Materials for Acoustically Induced Nonlinear Optics. Organometallics 2005, 24, 687-695.

(36) Colombo, A.; Nisic, F.; Dragonetti, C.; Marinotto, D.; Oliveri, I. P.; Righetto, S.; Lobello, M. G.; De Angelis, F. Unexpectedly High Second-Order Nonlinear Optical Properties of Simple $\mathrm{Ru}$ and $\mathrm{Pt}$ Alkynyl Complexes as an Analytical Springboard for NLO-Active Polymer Films. Chem. Commun. 2014, 50, 7986-7989.

(37) Dragonetti, C.; Colombo, A.; Marinotto, D.; Righetto, S.; Roberto, D.; Valore, A.; Escadeillas, M.; Guerchais, V.; Le Bozec, H.; Boucekkine, A.; Latouche, C. Functionalized Styryl Iridium(III) Complexes as Active Second-Order NLO Chromophores and Building Blocks for SHG Polymeric Films. J. Organomet. Chem. 2014, 751, $568-572$.

(38) Innocenzi, P.; Lebeau, B. Organic-Inorganic Hybrid Materials for Non-Linear Optics. J. Mater. Chem. 2005, 15, 3821-3831.

(39) Chaumel, F.; Jiang, H.; Kakkar, A. Sol-Gel Materials for Second-Order Nonlinear Optics. Chem. Mater. 2001, 13, 3389-3395.

(40) Yzambart, G.; Fabre, B.; Roisnel, T.; Dorcet, V.; Ababou-Girard, S.; Meriadec, C.; Lorcy, D. Assembly of Platinum Diimine Dithiolate Complexes Onto Hydrogen-Terminated Silicon Surfaces. Organometallics 2014, 33, 4766-4776.

(41) Balch, L.; Holm, R. H. Complete Electron-Transfer Series of the [M-N4] Type. J. Am. Chem. Soc. 1966, 88, 5201-5209. the method 1 reported in this reference was followed to prepare the $\left[\mathrm{Ni}(o-\mathrm{phen})_{2}\right]$ complex.

(42) Sellmann, D.; Binder, H.; Haussinger, D.; Heinemann, F. W.; Sutter, J. Transition Metal Complexes with Sulfur Ligands Part CXLIV. Square Planar Nickel Complexes with NiS4 Cores in Three Different Oxidation States: Synthesis, X-Ray Structural, and Spectroscopic Studies. Inorg. Chim. Acta 2000, 302, 829-836.

(43) Agilent Technologies, CrysAlisPro, 2011, Agilent Technologies UK Ltd., Oxford, U.K..

(44) Sheldrick, G. M. A Short History of SHELX. Acta Crystallogr., Sect. A: Found. Crystallogr. 2008, 64, 112-122.

(45) Palatinus, L.; Chapuis, G. SUPERFLIP-A Computer Program for the Solution of Crystal Structures by Charge Flipping in Arbitrary Dimensions. J. Appl. Crystallogr. 2007, 40, 786-790.

(46) Farrugia, L. J. ORTEP-3 for windows - A Version of ORTEP-III with a Graphical User Interface (GUI). J. Appl. Crystallogr. 1997, 30, 565 .

(47) Macrae, F.; Bruno, I. J.; Chisholm, J. A.; Edgington, P. R.; McCabe, P.; Pidcock, E.; Rodriguez-Monge, L.; Taylor, R.; van de Streek, J.; Wood, P. A. Mercury CSD 2.0-New Features for the Visualization and Investigation of Crystal Structures. J. Appl. Crystallogr. 2008, 41, 466-470.

(48) Parr, R. G.; Yang, W. Density Functional Theory of Atoms and Molecules; Oxford University Press: Oxford, U.K., 1989.

(49) Frisch, M. J.; Trucks, G. W.; Schlegel, H. B.; Scuseria, G. E.; Robb, M. A.; Cheeseman, J. R.; Scalmani, G.; Barone, V.; Mennucci, B.; Petersson, G. A.; et al. Gaussian 09, revision D.01; Gaussian, Inc.: Wallingford, CT, 2009.

(50) Becke, D. Density-Functional Thermochemistry. III. The Role of Exact Exchange. J. Chem. Phys. 1993, 98, 5648-5652.

(51) Lee, C.; Yang, W.; Parr, R. G. Development of the Colle-Salvetti Correlation-Energy Formula into a Functional of the Electron Density. Phys. Rev. B: Condens. Matter Mater. Phys. 1988, 37, 785-789.

(52) Yanai, T.; Tew, D. P.; Handy, N. C. A New Hybrid ExchangeCorrelation Functional Using the Coulomb-Attenuating Method (CAM-B3LYP). Chem. Phys. Lett. 2004, 393, 51-57.

(53) Adamo, C.; Barone, V. Toward Reliable Density Functional Methods Without Adjustable Parameters: The PBE0 Model. J. Chem. Phys. 1999, 110, 6158-6170.
(54) Krishnan, R.; Binkley, J. S.; Seeger, R.; Pople, J. A. SelfConsistent Molecular Orbital Methods. XX. A Basis Set for Correlated Wave Functions. J. Chem. Phys. 1980, 72, 650-654.

(55) McLean, A. D.; Chandler, G. S. Contracted Gaussian Basis Sets for Molecular Calculations. I. Second Row Atoms, $Z=11-18$. J. Chem. Phys. 1980, 72, 5639-5648.

(56) Cariati, E.; Pizzotti, M.; Roberto, D.; Tessore, F.; Ugo, R. Coordination and Organometallic Compounds and Inorganic-Organic Hybrid Crystalline Materials for Second-Order Non-Linear Optics. Coord. Chem. Rev. 2006, 250, 1210-1233 and references therein..

(57) Willetts, A.; Rice, J. E.; Burland, D. M.; Shelton, D. P. Problems in the Comparison of Theoretical and Experimental Hyperpolarizabilities. J. Chem. Phys. 1992, 97, 7590-7599.

(58) Kurtz, S. K.; Perry, T. T. A Powder Technique for the Evaluation of Nonlinear Optical Materials. J. Appl. Phys. 1968, 39, $3798-3813$.

(59) Cariati, E.; Macchi, R.; Roberto, D.; Ugo, R.; Galli, S.; Casati, N.; Macchi, P.; Sironi, A.; Bogani, L.; Caneschi, A.; Gatteschi, D. Polyfunctional Inorganic-Organic Hybrid Materials: An Unusual Kind of NLO Active Layered Mixed Metal Oxalates with Tunable Magnetic Properties and Very Large Second Harmonic Generation. J. Am. Chem. Soc. 2007, 129, 9410-9420.

(60) Heesink, G. J. T.; van Hulst, N. F.; Bölger, B.; Kelderman, E.; Engbersen, J. F. J.; Verboom, W.; Reinhoudt, D. N. Novel Calixarenes in Thin Films for Efficient Second Harmonic Generation. Appl. Phys. Lett. 1993, 62, 2015-2017.

(61) Boixel, J.; Guerchais, V.; Le Bozec, H.; Jacquemin, D.; Amar, A.; Boucekkine, A.; Colombo, A.; Dragonetti, C.; Marinotto, D.; Roberto, D.; Righetto, S.; De Angelis, R. Second-Order NLO Switches from Molecules to Polymer Films Based on Photochromic Cyclometalated Platinum(II) Complexes. J. Am. Chem. Soc. 2014, 136, 5367-5375.

(62) Hall, G. S.; Soderberg, R. H. The Crystal and Molecular Structure of Bis(o-phenylenediamino)nickel, $\mathrm{Ni}\left[\mathrm{C}_{6} \mathrm{H}_{4}(\mathrm{NH})_{2}\right]_{2}$. Inorg. Chem. 1968, 7, 2300-2303.

(63) Mrkvova, K.; Kameníček, J.; Sindelàr, Z.; Kvitek, L.; Mrozinski, J.; Nahorska, M.; Zàk, Z. Synthesis, Properties, and Crystal Structures of $\mathrm{R}\left[\mathrm{M}^{\mathrm{III}}(\mathrm{bdt})_{2}\right]$ Complexes $(\mathrm{M}=\mathrm{Ni}, \mathrm{Co}, \mathrm{Cu})$. Transition Met. Chem. 2004, 29, 238-244.

(64) Kameníček, J.; Herich, P.; Kuča, K.; Jun, D.; Olšovský, M. Planar $\mathrm{Ni}$ (II) 1,2-Dithiolenes Involving Bidentate N-Donor Ligands. J. Coord. Chem. 2008, 61, 3525-3533.

(65) Stalhandske, C. 1,2-Diaminobenzene, $\mathrm{C}_{6} \mathrm{H}_{4}\left(\mathrm{NH}_{2}\right)_{2}$. Cryst. Struct. Commun. 1981, 10, 1081-1086.

(66) Makedonas, C.; Mitsopoulou, C. Tuning the Properties of $\mathrm{M}$ (diimine) (dithiolate) Complexes-The Role of the Metal and Solvent Effect. A Combined Experimental, DFT and TDDFT Study. Inorg. Chim. Acta 2007, 360, 3997-4009.

(67) Noro, S.; Chang, H.-C.; Takenobu, T.; Murayama, Y.; Kanbara, T.; Aoyama, T.; Sassa, T.; Wada, T.; Tanaka, D.; Kitagawa, S.; et al. Metal-Organic Thin-Film Transistor (MOTFT) Based on a Bis(odiiminobenzosemiquinonate)nickel(II) Complex. J. Am. Chem. Soc. 2005, 127, 10012-10013 In order to make them directly comparable to our data, the $\mathrm{CV}$ values reported in the reference have been converted vs the $\mathrm{Ag} / \mathrm{AgCl}$ couple, accordingly to the half-wave potential for ferrocene/ferrocenium couple reported in the Experimental section..

(68) Miller, R. T.; Dance, G. I. Reactions of Dithiolene Complexes with Amines. II. The Formation and Properties of Mixed-Ligand Dithiolene $\alpha$-Diimine Complexes of Nickel. J. Am. Chem. Soc. 1973, 95, 6970-6979 The CV measurements reported in this reference are done in $\mathrm{CH}_{3} \mathrm{CN}$; furthermore, for the same reasons explained in the, the reported values have been converted from vs SCE to vs the $\mathrm{Ag} /$ AgCl couple..

(69) Cummings, S. D.; Eisenberg, R. Tuning the Excited-State Properties of Platinum(II) Diamine-Dithiolate Complexes. J. Am. Chem. Soc. 1996, 118, 1949-1960.

(70) Reichardt, C. Solvatochromic Dyes as Solvent Polarity Indicators. Chem. Rev. 1994, 94, 2319-2358. 
(71) Oudar, J. Optical Nonlinearities of Conjugated Molecules. Stilbene Derivatives and Highly Polar Aromatic Compounds. J. Chem. Phys. 1977, 67, 446-457.

(72) Oudar, J.; Chemla, D. S. Hyperpolarizabilities of the Nitroanilines and their Relations to the Excited State Dipole Moment. J. Chem. Phys. 1977, 66, 2664-2668.

(73) Bruni, S.; Cariati, E.; Cariati, F.; Porta, F. A.; Quici, S.; Roberto, D. Determination of the Quadratic Hyperpolarizability of Trans-4-[4(dimethylamino)styryl]pyridine and 5-Dimethylamino-1,10-Phenanthroline from Solvatochromism of Absorption and Fluorescence Spectra: A Comparison with the Electric-Field-Induced SecondHarmonic Generation Technique. Spectrochim. Acta, Part A 2001, 57, 1417-1426.

(74) Moylan, C. R.; Twieg, R. J.; Lee, V. Y.; Swanson, S. A.; Betterton, K. M.; Miller, R. D. Nonlinear Optical Chromophores with Large Hyperpolarizabilities and Enhanced Thermal Stabilities. J. Am. Chem. Soc. 1993, 115, 12599-12600.

(75) Curreli, S.; Deplano, P.; Faulmann, C.; Ienco, A.; Mealli, C.; Mercuri, M. L.; Pilia, L.; Pintus, G.; Serpe, A.; Trogu, E. F. Electronic Factors Affecting Second-Order NLO Properties: Case Study of Four Different Push-Pull Bis-Dithiolene Nickel Complexes. Inorg. Chem. 2004, 43, 5069-5079.

(76) Deplano, P.; Mercuri, M. L.; Serpe, A.; Pilia, L. The Chemistry of Metal Enolates; Zabicky, J., Ed.; Wiley\& Sons, Ltd: Chichester, U.K., 2009; Chapter 16, pp 879-928.

(77) Hampsch, H. L.; Torkelson, J. M.; Bethke, S. J.; Grubb, S. G. Second Harmonic Generation in Corona Poled, Doped Polymer Films as a Function of Corona Processing. J. Appl. Phys. 1990, 67, 10371041.

(78) Hampsch, H. L.; Yang, J.; Wong, G. K.; Torkelson, J. M. Dopant Orientation Dynamics in Doped Second-Order Nonlinear Optical Amorphous Polymers. 2. Effects of Physical Aging on Poled Films. Macromolecules 1990, 23, 3648-3654.

(79) Kelderman, E.; Heesink, G. J. T.; Derhaeg, L.; Verbiest, T.; Klaase, P. T. A.; Verboom, W.; Engbersen, J. F. J.; van Hulst, N. F.; Clays, K.; Persoons, A.; Reinhoudt, D. N. Highly Ordered Films of Neat Calix[4]arenes for Second Order Nonlinear Optics. Adv. Mater. 1993, 5, 925-930.

(80) Kenis, P. J. A.; Noordman, O. F. J.; Snellink-Ruël, B. H. M.; Engbersen, J. F. J.; van Hulst, N. F.; Reinhoudt, D. N. Molecular and Macroscopic Second Order Nonlinear Optical Properties of Calyx[4]arenes. Polym. Mater.: Sci. Eng. 1996, 75, 317-318.

(81) Page, R. H.; Jurich, M. C.; Reck, B.; Sen, A.; Twieg, R. J.; Swalen, J. D.; Bjorklund, G. C.; Wilson, C. G. Electrochromic and Optical Waveguide Studies of Corona-Poled Electro-Optic Polymer Films. J. Opt. Soc. Am. B 1990, 7, 1239-1250.

(82) Li, D.; Minami, N.; Ratner, M. A.; Ye, C.; Marks, T. J.; Yang, J.; Wong, G. K. Approaches to Polymeric Nonlinear Optical Materials. Theoretical and Synthetic Design Strategies. Synth. Met. 1989, 28, D585-D593. 